\title{
Protocol for the Isolation and Rapid Characterization of Foreign Debris in Pharmaceutical Products Using Light and Electron Microscopy
}

\author{
Brown, Richard S. ${ }^{1}$ \\ 1. MVA Scientific Consultants, Duluth, GA, USA.
}

Rapid analysis of unknown materials requires an experienced team of microscopists, information technologists (IT) and consistent laboratory procedures. The protocol consists of establishing communication between the manufacturer (customer) and the microscopist. A discussion between the customer and the microscopist occurs where any available background is shared to inform the microscopist about the conditions pertinent to the discovery and possible sources of the foreign debris. The microscopist will prepare and convey a procedure describing the analytical techniques used and prepare contingencies should the information developed drive the analysis in a different direction. Preliminary data will be sent to the manufacturer for the purpose of promoting discussion and exploring possibilities that may provide further insight into the source or sources of the foreign debris. The preliminary data should be sent as text in an email or using a secure file transfer protocol. The advantage of a secure file transfer system is that a record is produced documenting when and to whom the data Is delivered, not unlike tracking packages using FedEx or other package delivery systems. The discussions during the preliminary data review should be voice and/or video either as a conference call or a virtual meeting. Email is not an efficient system to transfer thoughts and ideas in real time, especially when the analysis is under a time constraint. Virtual meetings should be scheduled regularly in extended investigations to maintain communication and to keep the analysis on track.

\section{Protocol Summary}

1. Foreign debris discovered, contact lab for microscopical analysis.

2. Provide background data via telephone.

3. Send sample via traceable courier.

4. Lab notifies customer when sample is received, or not received when expected.

5. Lab documents condition upon receipt.

6. Lab isolates debris, initiates analyses.

7. The subsamples are analyzed using a combination of scanning electron microscopy-energy dispersive x-ray spectrometry (SEM-EDS), Fourier transform infrared microspectroscopy (FTIR) and polarized light microscopy (PLM). Additional analyses using analytical electron microscopy (AEM), fluorescence microscopy, microspectrophotometry and confocal Raman microscopy (CRM) may be performed on selected samples.

8. Preliminary data is conveyed to customer via traceable electronic data.

9. Customer reviews preliminary data.

10. Setup telephone conference and virtual meeting for lab to present preliminary data, and explain preliminary results.

11. Additional analyses initiated or final report issued.

The analysis of the same sample using multiple complimentary microscopical techniques provides rapid and reliable data to characterize foreign debris that occurs in pharmaceutical products. The data generated using multiple complimentary microscopical analyses provides a quality check on the results leading to reliable and consistent reporting. 


\section{References:}

[1] R. Brown, Investigation and Analysis of Medical Device Contamination; Practical Applications of Light and Electron Microscopy, Microscopy and Microanalysis (2014) p. 68.

[2] R. Brown, M. Miller and M. Zhou, "Foreign Particulate-Examination, Isolation and Analysis, September 17-18, 2015, PDA Research and Training Institute, Bethesda, MD.

[3] R. Brown, M. Miller and M. Zhou, "Pharmaceutical Foreign Particulate-Examination, Isolation and Analysis, June 5-6, 2014, InterMicro2014, McCrone Research Institute.
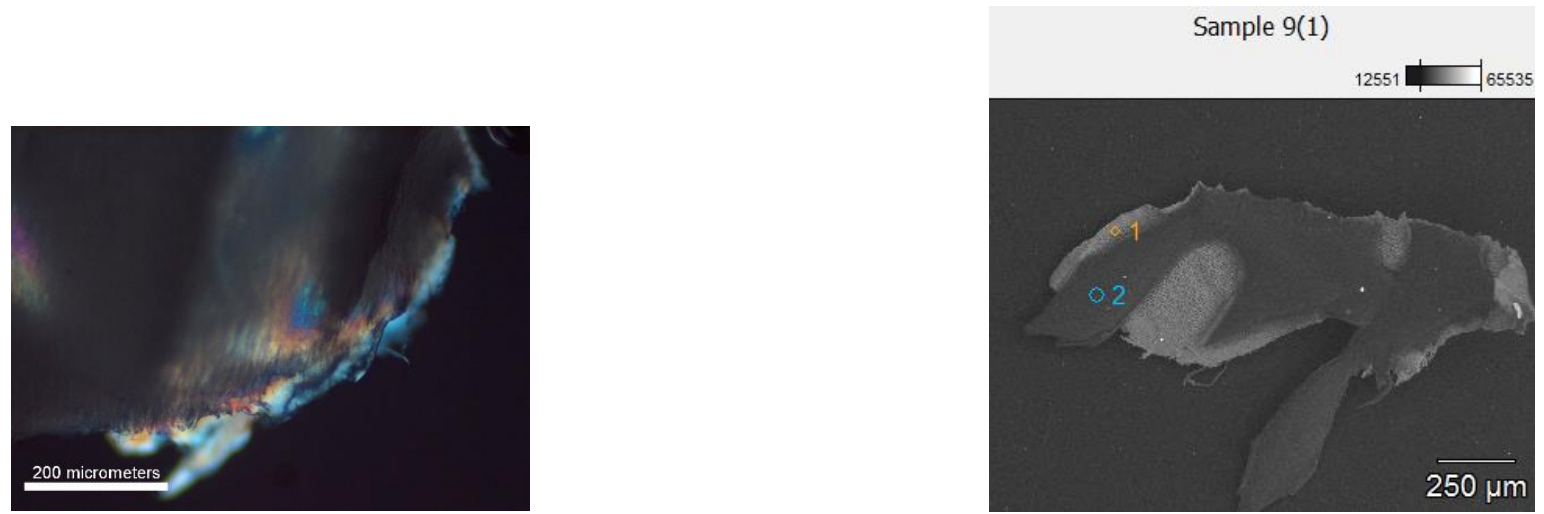

Figure 1. Two component filter media fragment. Above: images from left; PLM image with crossed polarizers and backscattered electron image. Below: EDS spectra and FTIR spectra from components 1 (fluoropolymer) and 2 (polypropylene).
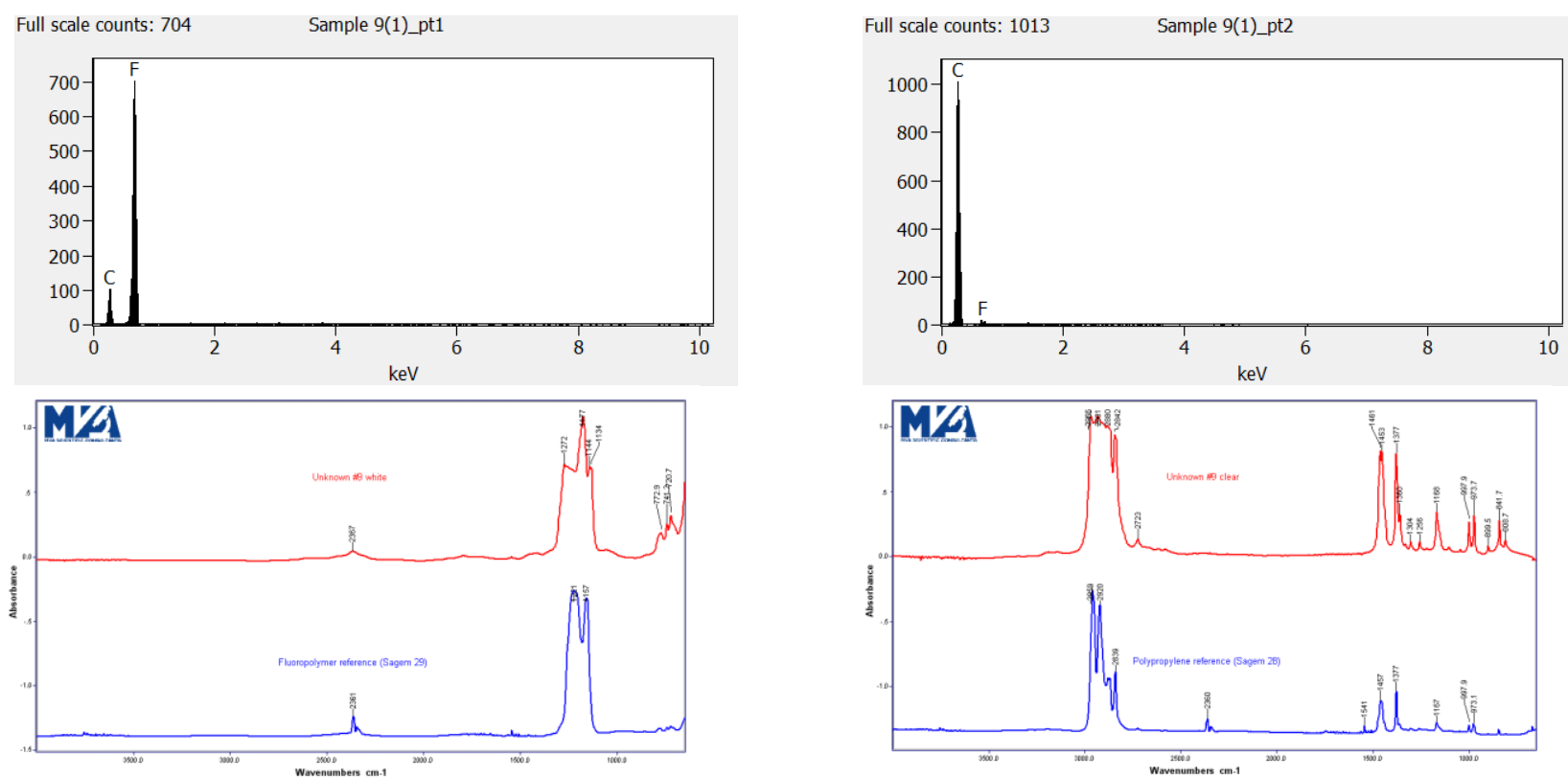